\title{
On the Cauchy transform vanishing outside a compact by
}

\section{Genadi LEVIN}

\section{Presented by Feliks PRZYTYCKI}

Summary. Motivated by a problem in holomorphic dynamics, we present a certain generalization of the celebrated F. and M. Riesz theorem.

1. Introduction. Given a finite complex measure $\nu$ with compact support on $\mathbb{C}$, let

$$
\hat{\nu}(z)=\int \frac{d \nu(w)}{w-z}
$$

be the Cauchy transform of $\nu$. For the following facts, see e.g. [12]: $\hat{\nu}$ is locally in $L^{1}(d x d y), \hat{\nu}$ exists almost everywhere on $\mathbb{C}$ and is holomorphic outside $\operatorname{supp}(\nu)$, and $\hat{\nu}(\infty)=0$. Moreover, if for an open set $U$ and an analytic function $h$ on $U, h=\hat{\nu} d x d y$-almost everywhere on $U$, then $|\nu|(U)=0$.

The F. and M. Riesz theorem asserts that, given a measure $\nu$ on the unit circle $S^{1}$, if $\int_{S^{1}} w^{n} d \nu(w)=0$ for all $n=1,2, \ldots$, in other words, if the Cauchy transform $\hat{\nu}$ of $\nu$ vanishes outside the closed unit disk, then $\nu$ is absolutely continuous with respect to the Lebesgue measure on $S^{1}$. Theorem 1 below is a generalization of this theorem to unions of pairwise disjoint bounded finitely connected domains.

We use the following notations and terminology. Given a compact subset $K$ of the plane, $A(K)$ is the algebra of all continuous functions on $K$ which are analytic in the interior of $K$, and $R(K)$ is the algebra of uniform limits on $K$ of rational functions with poles outside $K$ (= uniform limits on $K$ of

2020 Mathematics Subject Classification: Primary 30E10; Secondary 37F50.

Key words and phrases: Cauchy transform, F. and M. Riesz Theorem, rational approximation, rotation domain.

Received 14 December 2019; revised 6 May 2020.

Published online 15 June 2020. 
functions holomorphic on $K)$. If $R(K)=A(K)$, we call $K$ an $A$-compact, and if an A-compact $K$ is nowhere dense, then $K$ is a $C$-compact $(\mathrm{C}=$ continuous since in this case $A(K)=C(K)$, the set of all continuous functions on $K)$. Given an open bounded set $U$, let $A(U)$ be the algebra of all analytic functions in $U$ which extend continuously to $\bar{U}$.

Necessary and sufficient conditions for a compact in the plane to be an A- or a C-compact are given by Vitushkin [22]. Here are some sufficient conditions [10], [22]: $K$ is a C-compact if the area of $K$ is zero; $K$ is a Ccompact (respectively, an A-compact) if every point of $K$ (respectively, every point of $\partial K$ ) belongs to the boundary of a component of the complement $\mathbb{C} \backslash K$. Moreover, the boundary of an A-compact is always a C-compact (see e.g. [10]), though not vice versa.

We would like to know about non-trivial measures $\nu$ supported on the boundary $\partial K$ of $K$ such that $\hat{\nu}=0$ outside $K$. It is well known [10] that $\hat{\nu}=0$ off $K$ if and only if $\int f d \nu=0$ for all $f \in R(K)$. This implies that if $K$ is nowhere dense, such a non-trivial measure $\nu$ exists if and only if $K$ is not a C-compact.

Let us formulate a question motivated by a problem in holomorphic dynamics. Given a nowhere dense compact $E$, let $V=\bigcup_{k} \Omega_{k}$ be the union of some non-empty collection $\left\{\Omega_{k}\right\}$ of bounded components of the complement $\mathbb{C} \backslash E$. The problem we are interested in is the following:

Describe the sets $V$ and $E$ for which any measure $\nu$ supported on $E$ and such that $\hat{\nu}=0$ in $\mathbb{C} \backslash(E \cup V)$ is in fact supported on $\bigcup_{k} \partial \Omega_{k}$ and absolutely continuous with respect to harmonic measures of $\Omega$.

We prove in Theorem 1 that this is the case if each component $\Omega_{k}$ is finitely connected without isolated points in its boundary and the following three condition hold: (I) $\left\{\Omega_{k}\right\}$ is a so-called D-collection, (II) $A(\bar{V})=A(V)$, (III) (i) $\bar{V}$ is an A-compact, (ii) $E$ is a C-compact.

Let us comment on (I)-(III) (see below for more details). In condition (I), $\left\{\Omega_{k}\right\}$ is said to be a D-collection if harmonic measures of different domains are mutually singular and for each domain $\Omega$ of the collection, a holomorphic homeomorphism from a bounded circular domain $\Delta$ onto $\Omega$ extends as a one-to-one map onto a subset of full $(\operatorname{arc})$ measure to $\partial \Delta$. Condition (II) means that every continuous function on $\bar{V}$ which is holomorphic in $V$ is in fact holomorphic in the interior of $\bar{V}$. This is obviously the case if $\operatorname{Int}(\bar{V})=V$. In condition (III), (ii) along with $\hat{\nu}=0$ off $E \cup V$ implies that $\nu=0$ on $E \backslash \partial V$ (see Lemma 2.1), i.e., in fact $\nu$ is supported on $\partial V$. Note that (III) holds, for example, if $\mathbb{C} \backslash E$ has finitely many components.

If $E=S^{1}$, the unit circle, and $V=\mathbb{D}$, the unit disk, then (I)-(III) are satisfied and we recover the F. and M. Riesz theorem. 
All conditions of Theorem 1 turn out to be essential: the conclusion about the measure $\nu$ breaks down in general if one of the conditions (I), (II) or (III) does not hold; see Proposition 1 along with Examples 1 , 2 .

There also exists an abstract F. and M. Riesz theorem: see [10, Theorem 7.6]. It would be interesting to derive Theorem 1 from that result. The reason we proved Theorem 1 was to apply it to a problem in holomorphic dynamics: see Corollary 2.1 and [18.

After completing this note (arXiv:1911.05336, Nov 13, 2019) we found Erret Bishop's paper [4] and its sequel [5]. The main Theorem 3 of [5] is a particular case of our Theorem 1 in the case when the boundary $E$ of $E \cup V$ is equal to the boundary of the unbounded component of the complement to $E \cup V$ (it is fairly easy to see that this implies that all conditions (I)-(III) hold). In the concluding Remark III of [5] E. Bishop asks whether the analog of his Theorem 3 holds in a more general setting noting that "this seems to be a difficult question" and that it is clear that some extra hypotheses are necessary. Our Theorem 1 thus partially answers this question.

For another line of development of the F. and M. Riesz theorem and [4]-[5], see more recent papers [15], [16] by Dmitry Khavinson. Our Theorem 1 is close in spirit to [16, Theorem 1]. While we study measures on $\partial X$ for a compact $X$ by uniformizing components of the interior of $X$, in [15], [16] measures on $\partial X$ are approximated from outside $X$. Theorem 1 of [16] essentially states that given a compact $X$ for which the Dirichlet problem is always solvable, every measure $\mu$ on $\partial X$ such that $\hat{\mu}=0$ off $X$ is a weak-* limit of $\left\{\mu_{n}\right\}$ with $\left\|\mu_{n}\right\| \leq\|\mu\|$ and $d \mu_{n}=\left.f_{n}(z) d z\right|_{\partial X_{n}}$, for any sequence $X_{n}$ ( $\left.\supset X\right)$ of compacts decreasing to $X$ with analytic boundaries and some $f_{n} \in R\left(X_{n}\right)$.

Let us remark finally that we try to keep the proofs as self-contained and elementary as possible.

\section{Statements}

2.1. Main result and (counter-)examples. Unless stated otherwise, all measures considered are assumed to be complex and finite. Given two measures $\lambda$ and $\mu$ where $\mu$ is positive we write $\lambda \ll \mu$ if $\lambda$ is absolutely continuous with respect to $\mu$, and $\lambda_{1} \perp \lambda_{2}$ for two mutually singular measures $\lambda_{1}, \lambda_{2}$. Given a bounded plane domain $\Omega$ (i.e., a connected open subset of the plane) let $\omega_{\Omega}$ denote the harmonic measure on $\partial \Omega$ with respect to a fixed point in $\Omega$.

Recall that a domain of the plane is circular if its boundary consists of a finite number of disjoint circles. An example is the unit disk $\mathbb{D}$. Given a finitely connected bounded domain $\Omega$ whose boundary contains no isolated points, it is a classical result that there exist a bounded circular domain $\Delta_{\Omega}$ 
and a conformal homeomorphism

$$
\psi_{\Omega}: \Delta_{\Omega} \rightarrow \Omega
$$

By the Fatou theorem on radial limits, for (Lebesgue) almost every point $w \in \partial \Delta_{\Omega}$, the radial boundary value $\psi_{\Omega}(w)$ is defined.

Definition 2.1 (cf. [13], [1]). Given a (finite or infinite) collection $\left\{\Omega_{i}\right\}$ of pairwise disjoint finitely connected domains without isolated points on their boundaries, we call $\left\{\Omega_{i}\right\}$ a D-collection $(\mathrm{D}=$ Davie, see [7]) if each $\psi_{\Omega_{i}}$ extends radially as a one-to-one map on a subset of full measure of the boundary of $\Delta_{\Omega_{i}}$, and $\omega_{\Omega_{i}} \perp \omega_{\Omega_{j}}$ for $i \neq j$.

Our main result is

THEOREM 1. Let $V$ be a bounded open set such that each component of $V$ is finitely connected without isolated boundary points. Let $\left\{\Omega_{i}, \kappa_{i}\right\}_{i=1}^{N}$, $1 \leq N \leq \infty$, be a set of couples where $\left\{\Omega_{i}\right\}_{i=1}^{N}$ is the collection of all components of $V$ and each $\kappa_{i}$ is a holomorphic function on $\Omega_{i}$. For each $i$, fix a uniformization $\psi_{\Omega_{i}}: \Delta_{\Omega_{i}} \rightarrow \Omega_{i}$. Let $E \subset \mathbb{C}$ be a compact set without interior points such that $E \subset \mathbb{C} \backslash V$ and $E \supset \partial V$.

(P1) Assume that

(I) $\left\{\Omega_{i}\right\}_{i=1}^{N}$ is a D-collection,

(II) $A(V)=A(\bar{V})$,

(III) (i) $\bar{V}$ is an $A$-compact, (ii) $E$ is a $C$-compact.

Then (a) implies (b) where

(a) there exists a measure $\nu$ supported in $E$ such that

$$
\hat{\nu}(z)= \begin{cases}\kappa_{i}(z) & \text { if } z \in \Omega_{i}, \text { for each } i, \\ 0 & \text { if } z \in \mathbb{C} \backslash(E \cup V),\end{cases}
$$

(b) for every $i$,

$$
\left\|\kappa_{i}\right\|:=\limsup _{\epsilon \rightarrow 0} \int_{\partial \Omega_{i, \epsilon}}\left|\kappa_{i}(z)\right||d z|<\infty
$$

where $\Omega_{i, \epsilon}=\psi_{\Omega_{i}}\left(\left\{w: \operatorname{dist}\left(w, \partial \Delta_{\Omega_{i}}\right)>\epsilon\right\}\right)$. Moreover, (b1)

$$
\sum_{i=1}^{N}\left\|\kappa_{i}\right\|<\infty
$$

(b2) the following representation holds:

$$
\nu=\sum_{i=1}^{N} \nu_{i}
$$


where $\left\{\nu_{i}\right\}_{i=1}^{N}$ are pairwise mutually singular measures, $\nu_{i}$ is a measure on $\partial \Omega_{i}$ such that $\nu_{i} \ll \omega_{\Omega_{i}}$, and $\left\|\kappa_{i}\right\|=\left\|\nu_{i}\right\|$, the total variation of $\nu_{i}$. In particular, $\nu$ has no atoms.

(P2) Conversely, assume that (2)-(3) hold. Then there exists a measure $\nu$ supported on $\bigcup_{k=1}^{N} \partial \Omega_{k}$ such that

$$
\hat{\nu}(z)= \begin{cases}\kappa_{i}(z) & \text { if } z \in \Omega_{i} \text { for each } i \\ 0 & \text { if } z \in \mathbb{C} \backslash \bar{V}\end{cases}
$$

Moreover, if harmonic measures on different $\Omega_{k}$ are mutually singular, then $\nu$ admits a representation (4) as in (b2).

Notice that if $E=\partial V$ then (III)(i) implies (III)(ii).

Applying Theorem 1 with $E=\partial V$ and $\kappa_{i}=1$ for all $i$ we get an answer to [12, Problem 4.2, p. 55] for sets $V$ that satisfy conditions (I)-(III): Let $V=\bigcup_{k} \Omega_{k}$ be a bounded open set such that its components $\left\{\Omega_{k}\right\}$ form a D-collection and $A(V)=A(\bar{V})=R(\bar{V})$. Then there is a measure $\mu$ on $\partial V$ such that $\hat{\mu}=1$ in $V$ and $\hat{\mu}=0$ off $\bar{V}$ if and only if for each $k$ the linear measure $\Lambda\left(\partial \Omega_{k}\right)$ of $\partial \Omega_{k}$ is finite and $\sum_{k} \Lambda\left(\partial \Omega_{k}\right)<\infty$.

Given a measure supported on a compact in the closed unit disk $\overline{\mathbb{D}}$, the unit circle can be used as a "screen" to kill the Cauchy transform of this measure off $\overline{\mathbb{D}}$ :

ExAmple 1 (A. Volberg). Let $V=\mathbb{D} \backslash K$ where $\mathbb{D}=\{|z|<1\}$ and $K=$ $[0,1]$. Let $\nu_{K}$ be a measure supported in $K$. Assume that $\hat{\nu}_{K}\left(e^{i t}\right) \in L^{1}(0,2 \pi)$. On the unit circle $S^{1}=\{|z|=1\}$ with the one-dimensional Lebesgue measure (i.e., the arc length measure $d t, 0 \leq t \leq 2 \pi$ ), we define a new measure $\nu_{c}$ so that $d \nu_{c}\left(e^{i t}\right)=h_{c} d t$ with density

$$
h_{c}\left(e^{i t}\right)=\frac{1}{2 \pi} e^{i t} \hat{\nu}_{K}\left(e^{i t}\right)
$$

Let $\nu$ be a (finite) measure on $\partial V$ which is defined as follows: $\nu=\nu_{K}$ on $K$ and $\nu=\nu_{c}$ on $S^{1}$. Then

$$
\hat{\nu}(z)= \begin{cases}\hat{\nu}_{K}(z) & \text { if } z \in V \\ 0 & \text { if } z \in \mathbb{C} \backslash \bar{V}\end{cases}
$$

Indeed, for $z \in \mathbb{C} \backslash\left(K \cup S^{1}\right)$,

$$
\hat{\nu}_{c}(z)=\int_{S^{1}} \frac{d \nu_{c}(w)}{w-z}=\int_{K} \frac{d \nu_{K}(u)}{u-z} \int_{S^{1}} \frac{d w}{2 \pi i(w-z)(u-w)}
$$

where the inner integral is equal to -1 for $|z|>1$ and 0 for $z \in \mathbb{D} \backslash K$. The same calculation, hence (6) as well, hold if $K \subset \overline{\mathbb{D}}$ is any compact such that the length of $K \cap \partial \mathbb{D}$ is zero and $\nu_{K}$ is any measure on $K$ such that $\hat{\nu}_{K}\left(e^{i t}\right) \in L^{1}(0,2 \pi)$. 
Proposition 1. Conditions (I)-(III) of Theorem 1 are necessary for its conclusion: there exist open sets $V$ and measures $\nu$ supported on $E:=\partial V$ such that $V$ is simply connected, $\hat{\nu}=0$ off $\bar{V}$ and $\nu$ has atoms (so is not absolutely continuous with respect to harmonic measure on $\partial V$ ) while, in the notations of Theorem 1, for the sets $V$ and $E$ one and only one of the conditions (I), (II), (III) breaks down.

Proof. Let $K$ be any nowhere dense compact as in Example 1 such that $V=\mathbb{D} \backslash K$ is simply connected. Let $E=K \cup S^{1}=\partial V$. Since $\bar{V}=\overline{\mathbb{D}}$, $\bar{V}$ is an A-compact and $E=\partial V$ is a C-compact, so condition (III) holds. On the other hand, taking $\nu_{K}$ to be a discrete measure with $\operatorname{supp}\left(\nu_{K}\right)=K$ we see that the conclusion of Theorem 1 does not hold. This means that (I) or (II) has to break down. In particular, for $K=[0,1]$, condition (I) does not hold while (II) does, so condition (I) is necessary indeed. As for the necessity of (II), we choose $K=J$ where $J$ is a Jordan arc such that $V=\mathbb{D} \backslash J$ is a simply connected domain which satisfies (I), i.e., the Riemann map $\psi_{V}: \mathbb{D} \rightarrow V$ extends to a one-to-one map on a set of a full (arc) measure on $S^{1}$. Hence, (II) cannot hold in this case (this also follows directly from [6. Theorem 1']; see moreover [2], [3]). The existence of such a Jordan arc $J$ follows from Browder and Wermer [6]. Indeed, in [6] an example of a Jordan arc $J$ is constructed such that the Riemann map $h: \mathbb{C} \backslash \overline{\mathbb{D}} \rightarrow \mathbb{C} \backslash J$ with $h(\infty)=\infty$ extends one-to-one on a set of full Lebesgue measure on $S^{1}$. Then it is easy to see that if we take $J \subset \overline{\mathbb{D}}$ with $J \cap S^{1}=\{1\}$, then $V=\mathbb{D} \backslash J$ satisfies (I). [Proof: since $V \subset \mathbb{C} \backslash J, h^{-1}(V)$ is a well defined simply connected bounded domain with pairwise analytic boundary; hence, if $\beta: \mathbb{D} \rightarrow h^{-1}(V)$ is a Riemann map then $\psi_{V}:=h \circ \beta: \mathbb{D} \rightarrow V$ extends to a one-to-one-map on a set of full measure on $S^{1}$.]

That (III) is necessary as well is shown by the following example.

Example 2. Let $J$ be the Jordan arc as in [6] (see the proof of Proposition 11). One can assume that $J \subset \mathbb{D} \cup\{1\}$ and the endpoints of $J$ are 0 and 1 . Let $\left\{D_{j}\right\}_{j=1}^{\infty}$ be a collection of open disks in $\mathbb{D} \backslash J$ with pairwise disjoint closures such that each $D_{j}$ touches $J$ at precisely one point which is neither 0 nor 1 , and the set of all such points is dense in $J$ and

$$
\sum_{j=1}^{\infty} \frac{r_{j}}{d_{j}}<\infty
$$

where $r_{j}$ is the radius of $D_{j}$ and $d_{j}$ is the distance between 0 and $D_{j}$. (It is not difficult to show that such a choice of disks is possible.) Define $V=$ $\mathbb{D} \backslash\left(J \cup \bigcup_{j=1}^{\infty} \bar{D}_{j}\right)$ and $E=\partial V=S^{1} \cup J \cup \bigcup_{j=1}^{\infty} S_{j}$ where $S_{j}=\partial D_{j}$. Since there are non-constant continuous functions on the Riemann sphere which are holomorphic in $\mathbb{C} \backslash J$, by [9], [10] the compact $\bar{V}=\overline{\mathbb{D}} \backslash \bigcup_{j=1}^{\infty} D_{j}$ is not an A-compact, i.e., $R(\bar{V}) \neq A(\bar{V})$. 
Now we define a measure $\nu$ on $E$ as follows. Let $\nu=\delta_{0}+\sum_{j=0}^{\infty} \nu_{j}$ where $\delta_{0}$ is the Dirac measure at $0, d \nu_{0}(w)=\hat{\delta}_{0}(w) d w, w \in S^{1}$, is a measure on $S^{1}$ and, for each $j, d \nu_{j}(w)=-\hat{\delta}_{0}(w) d w, w \in S_{j}$, is a measure on $S_{j}$. Since for every $j \geq 0,\left\|\nu_{j}\right\| \leq 2 \pi r_{j} / d_{j}$, we have

$$
\|\nu\| \leq 1+2 \pi\left(1+\sum_{j=1} \frac{r_{j}}{d_{j}}\right)<\infty .
$$

Similar to Example 1, we get $\hat{\nu}=0$ off $\tilde{E}$ and $\hat{\nu}=\hat{\delta}_{0}$ in $V$. On the other hand, $\nu$ is not absolutely continuous with respect to harmonic measure of $V$ because $\nu$ has an atom at 0 . Notice that $V$ is simply connected and, for the sets $V, E$ as above, conditions (I), (II) of Theorem 1 hold but (III) does not: $\bar{V}$ is not an A-compact. Note that at the same time, $E=\partial V$ is a C-compact as every point of $E$ is at the boundary of the component $V$ of the complement to $E$.

2.2. Local removability of C-compacts. We need the following

\section{LEMMA 2.1.}

(a) Every closed subset of a $C$-compact is a $C$-compact.

(b) Let $K$ be a nowhere dense compact in $\mathbb{C}$ and $\mu$ a measure on $K$. Suppose that for a neighborhood $W$ of a point $x \in K, K \cap \bar{W}$ is a $C$-compact and $\hat{\mu}=0$ on $W \backslash K$. Then $\mu$ vanishes on $K \cap W$, i.e., $|\mu|(W)=0$.

Proof. (a) follows from the fact that any continuous function on a closed subset of a compact extends to a continuous function on the whole compact.

Let us prove (b). Let $U$ be the union of all components of $\mathbb{C} \backslash K$ that intersect $W$. Then $W \backslash K \subset U$ and $\hat{\mu}=0$ on $U$. One can assume that $\infty \in U$ as otherwise, for $M(z)=1 /\left(x_{0}-z\right)$ with some $x_{0} \in U$, we replace $K$ by $\tilde{K}=M(K)$ and $\mu$ by a measure $\tilde{\mu}$ on $\tilde{K}$ such that $d \tilde{\mu}(w)=w d \mu\left(x_{0}-1 / w\right)$. Thus $\infty \in U$.

Now, to get $|\mu|(W)=0$ it is enough to prove that for each $x \in K \cap W$ there is a neighborhood $W_{x} \subset W$ such that for all continuous functions $g$ with compact support in $W_{x}, \int g d \mu=0$. So fix $x \in K \cap W$ and choose $W_{x}=B(x, r)$ where $r>0$ is such that $B(x, 2 r) \subset W$. Let $g$ be a continuous function on $\mathbb{C}$ which is compactly supported in $B(x, r)$. Let $\hat{K}=\mathbb{C} \backslash U$. It is enough to prove that $g \in R(\hat{K})$.

Indeed, assume that there is a sequence of rational functions $R_{n}$ with poles outside $\hat{K}$ converging uniformly on $\hat{K}$ to $g$. Perturbing some of $R_{n}$ if necessary one can further assume that all $R_{n}$ have simple poles. If say $R_{n}(z)=P(z)+\sum_{j=1}^{m} \alpha_{j} /\left(z-b_{j}\right)$ where $P$ is a polynomial and all $b_{j}$ are in $U$, then $\int R_{n} d \mu=\int P d \mu+\sum_{j=1}^{m} \alpha_{j} \hat{\mu}\left(b_{j}\right)=0$ because $\hat{\mu}=0$ in $U$ and $\int z^{n} d \mu(z)=0$ for all $n>0$ (this is because $\hat{\mu}=0$ in a neighborhood of $\infty$ ). 
Then $\int g d \mu=\lim _{n} \int R_{n} d \mu=0$. It remains to show that $g \in R(\hat{K})$. We use Bishop's theorem [23, 11.8]): Given a compact $X \subset \mathbb{C}$ and a continuous function $f$ on $\mathbb{C}$, assume that for each $z \in X$ there is a closed neighborhood $B_{z}=\left\{|w-z| \leq \delta_{z}\right\}$ with $\delta_{z}>0$ such that $\left.f\right|_{X \cap B_{z}} \in R\left(X \cap B_{z}\right)$. Then $f \in R(X)$. Applying this to the compact $\hat{K}$ and the function $g$ shows that if $z \in \hat{K} \backslash \bar{B}(x, r)$, then, for $\delta_{z}=|z-x|-r>0,\left.g\right|_{\hat{K} \cap B_{z}}=0 \in R\left(\hat{K} \cap B_{z}\right)$. On the other hand, for $z \in \bar{B}(x, r)$ and $\delta_{z}=r,\left.g\right|_{\hat{K} \cap B_{z}} \in R\left(\hat{K} \cap B_{z}\right)$ because $\hat{K} \cap B_{z} \subset \hat{K} \cap \bar{B}(x, 2 r) \subset K \cap \bar{W}$ while $K \cap \bar{W}$ is a C-compact. Notice that $g \in R(\hat{K})$ also follows from Vitushkin's necessary and sufficient condition for a function to be in $R(X)$ (see [22]).

2.3. A particular case: rotation domains. Let $\left\{\Omega_{i}\right\}$ be a collection of pairwise disjoint Jordan domains such that $\bar{\Omega}_{i} \cap \bar{\Omega}_{j}$ is at most a single point for all $i \neq j$, and $V=\bigcup_{i} \Omega_{i}$ is bounded. Then obviously conditions (I)-(II) of Theorem 1 hold.

Here is a more interesting case which originates from holomorphic dynamics. Recall that a simply or doubly connected domain $A$ is called a rotation domain for a rational function $f$ of degree at least 2 if $f^{Q}: A \rightarrow A$ is a homeomorphism for some $Q \geq 1$ and is conjugate to an irrational rotation: for a conformal homeomorphism $\psi_{A}: \Delta_{A} \rightarrow A$ where $\Delta_{A}$ is either a round disk or a round annulus, the conjugate map $R:=\psi_{A}^{-1} \circ f^{Q} \circ \psi_{A}: \Delta_{A} \rightarrow \Delta_{A}$ is an irrational rotation of $\Delta_{A}$. ( $A$ is called a Siegel disk or a Herman ring depending on whether $\Delta_{A}$ is a disk or an annulus.)

Proposition 2. Let $\{A\}$ be a collection of different rotation domains of a rational function $f$, i.e., each $A$ is either a Siegel disk or a Herman ring. Then $\{A\}$ satisfies conditions (I)-(II) of Theorem 1, i.e.,

(1) for every $A$, there is a subset $\tilde{X}$ of $\partial \Delta_{A}$ of full Lebesgue (length) measure on which $\psi_{A}$ is one-to-one,

(2) for any two different rotation domains $A_{1}, A_{2}$, their harmonic measures $\omega_{A_{1}}$ and $\omega_{A_{2}}$ are mutually singular,

(3) if $V_{f}:=\bigcup_{A \in\{A\}} A$, then the interior of $\bar{V}_{f}$ is equal to $V_{f}$, and therefore $A\left(V_{f}\right)=A\left(\bar{V}_{f}\right)$.

Part (1) is an easy corollary of the following claim which is proved in [19. Theorem 2] for the Siegel disk and the proof holds with obvious modifications for the Herman ring:

LemMA 2.2. Let $A$ be a rotation domain of a rational function $f$ such that $f(A)=A$ and $\psi_{A}: \Delta_{A} \rightarrow A$ as above. There are only two cases possible:

(i) All radial limits $\psi_{A}(w)$ are different.

(ii) There is a point $a \in \partial A$ such that $f(a)=a$, and if $\psi_{A}\left(w_{1}\right)=\psi_{A}\left(w_{2}\right)$ for some $w_{1}, w_{2} \in \partial \Delta_{A}$ then $\psi_{A}\left(w_{1}\right)=\psi_{A}\left(w_{2}\right)=a$. 
Proof of Proposition 2. (1) follows at once from Lemma 2.2 and Riesz's uniqueness theorem for bounded analytic functions.

Let us prove (2). It is enough to show that the following is impossible: $\omega_{A_{1}}(Y)>0$ and $\omega_{A_{2}}(Y)>0$ for $Y=\overline{A_{1}} \cap \overline{A_{2}}$ where $Y$ is a subset of a component $L_{1}$ of $\partial A_{1}$ as well as a component $L_{2}$ of $\partial A_{2}$. So assume this is the case. First, since $f^{Q}\left(L_{i}\right)=L_{i}$ for some $Q>0$ and $i=1,2$, we have $f^{Q}(Y) \subset Y$. Secondly, by the Fatou theorem on radial limits, there is a set $\tilde{Y} \subset \partial \Delta_{A_{1}}$ of length $|\tilde{Y}|>0$ such that for all $w \in \tilde{Y}$, the radial limit $\psi_{A_{1}}(w)$ exists and is in $Y$. Moreover, since $f^{Q}(Y) \subset Y$, one can assume that $R(\tilde{Y}) \subset \tilde{Y}$ where $R=\psi_{A_{1}}^{-1} \circ f^{Q} \circ \psi_{A_{1}}: \Delta_{A_{1}} \rightarrow \Delta_{A_{1}}$ is an irrational rotation of $\Delta_{A_{1}}$. Since $|\tilde{Y}|>0$, we find that $\tilde{Y}$ has full measure. Then $Y$ is a closed subset of $L_{1}$ of full harmonic measure $\omega_{A_{1}}$, and hence $Y=L_{1}$. Since $\omega_{A_{2}}(Y)>0$, we have $Y=L_{2}$ as well, i.e., $L_{1}=L_{2}$. Taking now a small disk $B$ around some $x \in L_{1}=L_{2}$, we see that all iterates $f^{j Q} B, j \geq 0$, stay in $\overline{A_{1} \cup A_{2}}$, which is possible only if $x$ is in the Fatou set, a contradiction. Moreover, that the interior of $\overline{V_{f}}$ coincides with $V_{f}$ is also proved by a very similar argument.

Theorem 1 and Proposition 2 immediately imply

Corollary 2.1. Suppose $\mathcal{H}$ is a non-empty collection of bounded rotation domains of a rational function $f$. Let $V=\bigcup\{A: A \in \mathcal{H}\}, E \subset \mathbb{C} \backslash V$ a nowhere dense compact set such that $\partial V \subset E$, and $\nu$ a measure supported on $E$ such that $\hat{\nu}=0$ off $E \cup V$. If $E$ is a $C$-compact and $\bar{V}$ is an $A$-compact, then $\nu$ is, in fact, supported on $\partial V=\bigcup_{A \in \mathcal{H}} \partial A$ and, for each $A$, $\left.\nu\right|_{\partial A} \ll \omega_{A}$. In particular, $\nu$ is non-atomic. Moreover, the function $\hat{\nu} \circ \psi_{A}^{\prime}$ is in the $H^{1}$-Hardy space, i.e.,

$$
\limsup _{\epsilon \rightarrow 0} \int_{\left\{z \in \Delta_{A}: \operatorname{dist}(z, \partial A)=\epsilon\right\}}\left|\hat{\nu} \circ \psi_{A}^{\prime}(z)\right||d z|<\infty .
$$

Conjecturally, $\bar{V}=\bigcup\{\bar{A}: A \in \mathcal{H}\}$ is always an A-compact.

\section{Proof of Theorem 1}

3.1. Preparatory statements. The proof is heavily based on some general results, mainly from the 1960s (see Theorems 2 4). The first one is a consequence of the Hahn-Banach theorem and the Riesz representation theorem (see [12]):

TheOREM 2 (V. P. Havin). Let $F \subset \mathbb{C}$ be compact and let $g$ analytic on $\mathbb{C} \backslash F$ and $g(\infty)=0$. There is a measure $\nu$ on $F$ such that $g(z)=\hat{\nu}(z)$ for all $z \notin F$ if and only if there is $C_{g}$ such that for all functions $h$ which are analytic in a neighborhood of $F$,

$$
\left|T_{g}(h)\right| \leq C_{g}\|h\|_{F}
$$


where $\|h\|_{F}=\sup _{z \in F}|h(z)|$ and

$$
T_{g}(h)=-\frac{1}{2 \pi i} \int_{\partial U} g(z) h(z) d z
$$

where $U$ is any small enough neighborhood of $F$ such that $\partial U$ consists of finitely many analytic curves that surround $E$ in positive direction. When this is the case, we may take $C_{g}=\|\nu\|$, the total variation of $\nu$.

Theorem 3 (G. G. Tumarkin, [21]). Let $g$ be analytic in $\mathbb{C} \backslash S^{1}$ and $g(\infty)=0$. Then $g=\hat{\eta}$ for some measure $\eta\left(\right.$ with $\left.\operatorname{supp}(\eta) \subset S^{1}\right)$ if and only if

$$
\sup _{0<r<1} \int_{S^{1}}|g(r \theta)-g(\theta / r)||d \theta|<\infty .
$$

Comment 1. Notice the particular case when $g=0$ off $\overline{\mathbb{D}}$.

For completeness, we prove this statement for the direction we need. So let $g=\hat{\eta}$ for a measure $\eta$ on $S^{1}$. Given $\zeta=e^{i \theta}, w=e^{i t} \in S^{1}$ and $0<r<1$ we have

$$
\frac{1}{w-r \zeta}-\frac{1}{w-\zeta / r}=-\frac{\zeta\left(r^{2}-1\right)}{w \zeta(1-r \zeta / w)(r w / \zeta-1)}=e^{-i t} P_{r}(\theta-t)
$$

where $P_{r}(\theta-t)=\left(1-r^{2}\right) /\left(1+r^{2}-2 r \cos (\theta-t)\right)$ is the Poisson kernel. Therefore,

$$
\hat{\eta}(r \zeta)-\hat{\eta}(\zeta / r)=\int_{0}^{2 \pi} P_{r}(\theta-t) e^{-i t} d \eta\left(e^{i t}\right)
$$

and

$$
\begin{aligned}
\int_{S^{1}}|g(r \zeta)-g(\zeta / r)||d \zeta| & \leq \int_{0}^{2 \pi} d \theta \int_{0}^{2 \pi} P_{r}(\theta-t) d|\eta|\left(e^{i t}\right) \\
& \leq \int_{0}^{2 \pi} d|\eta|\left(e^{i t}\right) \int_{0}^{2 \pi} P_{r}(\theta-t) d \theta=2 \pi\|\eta\|
\end{aligned}
$$

where $\|\eta\|$ is the total variation of the measure $\eta$.

The next two statements are most probably well known too, though we are not aware of precise references and for completeness give the proofs (cf. [4]-5]). For the first one, recall the following definition. Let $D$ be a bounded or unbounded circular domain, i.e., $\partial D=S_{1} \cup \cdots \cup S_{p}$ where $S_{1}, \ldots, S_{p}$ are pairwise disjoint circles. The Hardy space $H^{1}(D)$ is the set of all holomorphic functions $F$ in $D$ with $F(\infty)=0$ if $\infty \in D$ such that

$$
\|F\|_{H^{1}(D)}:=\limsup _{S \in \mathcal{S}} \int_{S}|F(w)||d w|<\infty
$$


where $\mathcal{S}$ is the collection of all circles $S \subset D$ in a small neighborhood of $\partial D$ that are concentric with one of $S_{j}, j=1, \ldots, p$. It is well known (see e.g. [14]) that any $F \in H^{1}(D)$ has a non-tangential limit $F(w)$ at almost every $w \in \partial D$ with respect to the Lebesgue (arc) measure on $\partial D$. We also need the following representation for $F \in H^{1}(D)$ assuming $D$ is bounded and $S_{1}$ is the outer boundary of $D$ :

$$
F=F_{1}+\cdots+F_{p}
$$

where $F_{j} \in D_{j}, D_{1}$ is a bounded domain with boundary $S_{1}$, and $D_{j}, j=$ $2, \ldots, p$, is an unbounded domain with $\partial D_{j}=S_{j}$. This representation follows essentially from the Cauchy formula (see [14]). Note that each $F_{j}$ is holomorphic in a domain that contains all other $S_{k}, k \neq j$.

LEMma 3.1. Let $\Omega$ be a finitely connected bounded domain with no isolated points of the boundary and let $\kappa: \Omega \rightarrow \mathbb{C}$ be holomorphic. Assume that

$$
\|\kappa\|:=\limsup _{\epsilon \rightarrow 0} \int_{\partial \Omega_{\epsilon}}|\kappa(w)||d w|<\infty
$$

where $\Omega_{\epsilon}=\psi_{\Omega}\left(\left\{w: \operatorname{dist}\left(w, \partial \Delta_{\Omega}\right)>\epsilon\right\}\right)$. Then there is a measure $\nu^{\kappa}$ supported on $\partial \Omega$ such that $\hat{\nu}^{\kappa}(z)=\kappa(z)$ for $z \in \Omega$ and $\hat{\nu}^{\kappa}(z)=0$ for $z \notin \bar{\Omega}$. Furthermore, $\nu^{\kappa} \ll \omega_{\Omega}$ with $\left\|\nu^{\kappa}\right\|=\|\kappa\|$.

Proof. Choose $\epsilon_{n} \rightarrow 0$ and given $n$ define a measure $\nu_{n}$ on $\partial \Omega_{\epsilon_{n}}$ by $d \nu_{n}(z)=\frac{1}{2 \pi i} \kappa(z) d z$. By (8), $\sup _{n}\left\|\nu_{n}\right\|<\infty$. Let $\nu^{\kappa}$ be a weak* limit of the sequence $\left\{\nu_{n}\right\}$. By the Cauchy formula,

$$
\hat{\nu}^{\kappa}(z)=\lim _{n \rightarrow \infty} \frac{1}{2 \pi i} \int_{\partial \Omega_{\epsilon_{n}}} \frac{\kappa(\zeta) d \zeta}{\zeta-z}
$$

is equal to $\kappa(z)$ for $z \in \Omega$ and 0 outside $\bar{\Omega}$. Denote $\psi=\psi_{\Omega}, \Delta=\Delta_{\Omega}$, $\left.\Gamma_{n}=\left\{w: \operatorname{dist}(w, \partial \Delta)=\epsilon_{n}\right\}\right)$ and $\tilde{\kappa}=(\kappa \circ \psi) \psi^{\prime}$. Note that (8) is equivalent to $\tilde{\kappa} \in H^{1}(\Delta)$. Let $\psi(w)$ and $\tilde{\kappa}(w), w \in \partial \Delta$, denote also the corresponding limits (existing almost everywhere) of $\psi(u)$ and $\tilde{\kappa}(u)$ as $u \rightarrow w$ non-tangentially. We have to prove that $\nu^{\kappa} \ll \omega_{\Omega}$. For this, it is enough to show that for any continuous compactly supported function $h$ on $\mathbb{C}$,

$$
\int h d \nu^{\kappa}=\frac{1}{2 \pi i} \int_{\partial \Delta} h(\psi(w)) \tilde{\kappa}(w) d w .
$$

This would imply that $\nu^{\kappa} \ll \omega_{\Omega}$ and that $\left\|\nu_{\kappa}\right\|=\|\kappa\|$. One can check (9) separately for each component of $\partial \Delta$. Let us do this for the outer component $S_{1}$ of $\partial \Delta=S_{1} \cup \cdots \cup S_{p}$ (for the other components, the proof is the same with straightforward modifications). One can assume $S_{1}=S^{1}$, the unit circle. 
Since $\int h d \nu^{\kappa}=\lim _{n \rightarrow \infty} \int h d \nu_{n}$, we have to check that, for $r_{n}=1-\epsilon_{n}$,

$$
\lim _{n \rightarrow \infty} \frac{1}{2 \pi i} \int_{|w|=r_{n}} h(\psi(w)) \tilde{\kappa}(w) d w=\frac{1}{2 \pi i} \int_{|w|=1} h(\psi(w)) \tilde{\kappa}(w) d w .
$$

As $\tilde{\kappa} \in H^{1}(\Delta)$, let $\tilde{\kappa}=\sum_{j=1}^{p} \tilde{\kappa}_{j}$ be the corresponding representation as in (7). Then

$$
\int_{|w|=r_{n}} h(\psi(w)) \tilde{\kappa}(w) d w=\sum_{j=1}^{p} \int_{|w|=r_{n}} h(\psi(w)) \tilde{\kappa}_{j}(w) d w .
$$

Let $j>1$. Since $\tilde{\kappa}_{j}, j \neq 1$, is a holomorphic function in a domain that contains $S^{1}, h(\psi(w)) \tilde{\kappa}_{j}(w)$ is bounded in $\{r<|w|<1\}$ for some $r<1$, hence, one can apply the Lebesgue dominated convergence theorem:

$$
\lim _{n \rightarrow \infty} \int_{|w|=r_{n}} h(\psi(w)) \tilde{\kappa}_{j}(w) d w=\int_{|w|=1} h(\psi(w)) \tilde{\kappa}_{j}(w) d w .
$$

It remains to check that

$$
\lim _{n \rightarrow \infty} \int_{|w|=r_{n}} h(\psi(w)) \tilde{\kappa}_{1}(w) d w=\int_{|w|=1} h(\psi(w)) \tilde{\kappa}_{1}(w) d w .
$$

Here we have to use the fact that $\tilde{\kappa}_{1} \in H^{1}(\mathbb{D})$, the Hardy space in the unit disk. We have

$$
\begin{aligned}
\mid \int_{|w|=r_{n}} h(\psi(w)) \tilde{\kappa}_{1}(w) d w & -\int_{|w|=1} h(\psi(w)) \tilde{\kappa}_{1}(w) \mid \\
\leq & \int_{0}^{2 \pi} \mid r_{n} h\left(\psi\left(r_{n} e^{i t}\right)\right)-h\left(\psi\left(e^{i t}\right)|| \tilde{\kappa}_{1}\left(r_{n} e^{i t}\right) \mid d t\right. \\
& +\int_{0}^{2 \pi}\left|h\left(\psi\left(e^{i t}\right)\right)\right|\left|\tilde{\kappa}_{1}\left(r_{n} e^{i t}\right)-\tilde{\kappa}_{1}\left(e^{i t}\right)\right| d t \rightarrow 0 .
\end{aligned}
$$

Here are some details. Let $I_{n}$ be the first integral on the RHS, and $J_{n}$ the second one. Then

$$
J_{n} \leq \sup _{t \in[0,2 \pi]}\left|h\left(\psi\left(e^{i t}\right)\right)\right| \int_{0}^{2 \pi}\left|\tilde{\kappa}_{1}\left(r_{n} e^{i t}\right)-\tilde{\kappa}_{1}\left(e^{i t}\right)\right| d t \rightarrow 0
$$

because $\tilde{\kappa}_{1} \in H^{1}(\mathbb{D})$ [17, Ch. II, B, $2^{\circ}$. Let us prove that $\lim _{n} I_{n}=0$. Let $I:=\lim \sup _{n} I_{n}$. Since $r \psi\left(r e^{i t}\right) \rightarrow \psi\left(e^{i t}\right)$ as $r \rightarrow 1$ a.e. in $t$ and $h$ is continuous, for every $\sigma>0$ there is $E_{\sigma} \subset[0,2 \pi]$ such that $l\left(E_{\sigma}\right)>2 \pi-\sigma$ (where $l$ is the Lebesgue measure on $(0,2 \pi))$ and $r_{n} h\left(\psi\left(r_{n} e^{i t}\right)\right)-h\left(\psi\left(e^{i t}\right)\right)$ $\rightarrow 0$ uniformly in $t \in E_{\sigma}$. Since also $\tilde{\kappa}_{1} \in H^{1}(\mathbb{D})$,

$$
\lim _{n} \int_{E_{\sigma}} \mid r_{n} h\left(\psi\left(r_{n} e^{i t}\right)\right)-h\left(\psi\left(e^{i t}\right)|| \tilde{\kappa}_{1}\left(r_{n} e^{i t}\right) \mid d t=0 .\right.
$$


Hence,

$$
I \leq 2 \sup |h| \limsup \int_{F_{\sigma}}\left|\tilde{\kappa}_{1}\left(r_{n} e^{i t}\right)\right| d t
$$

where $F_{\sigma}=[0,2 \pi] \backslash E_{\sigma}$ so that $l\left(F_{\sigma}\right) \leq \sigma$. On the other hand,

$$
\begin{aligned}
\left|\int_{F_{\sigma}}\right| \tilde{\kappa}_{1}\left(r_{n} e^{i t}\right)\left|d t-\int_{F_{\sigma}}\right| \tilde{\kappa}_{1}\left(e^{i t}\right)|d t| & \leq \int_{0}^{2 \pi} \chi_{F_{\sigma}}\left|\tilde{\kappa}_{1}\left(r_{n} e^{i t}\right)-\tilde{\kappa}_{1}\left(e^{i t}\right)\right| d t \\
& \leq \int_{0}^{2 \pi}\left|\tilde{\kappa}_{1}\left(r_{n} e^{i t}\right)-\tilde{\kappa}_{1}\left(e^{i t}\right)\right| d t \rightarrow 0
\end{aligned}
$$

as $r \rightarrow 1$. Thus

$$
I \leq 2 \sup |h| \int_{F_{\sigma}}\left|\tilde{\kappa}_{1}\left(e^{i t}\right)\right| d t .
$$

By the absolute continuity of the Lebesgue integral, for every $\epsilon>0$ there is $\sigma>0$ such that for every $F_{\sigma} \subset[0,2 \pi]$ with $l\left(F_{\sigma}\right)<\sigma$,

$$
I \leq 2 \sup |h| \int_{F_{\sigma}}\left|\tilde{\kappa}_{1}\left(e^{i t}\right)\right| d t<2 \sup |h| \epsilon .
$$

As $\epsilon>0$ is arbitrary, $I=0$.

In the following, for a bounded function $h: X \rightarrow \mathbb{C}$, let

$$
\|h\|_{X}=\sup _{x \in X}|h(x)| .
$$

LEMMA 3.2. Let $g \not \equiv 0$ be analytic in a bounded circular domain $\Delta$. Assume that there is $C_{g}>0$ such that for every function h holomorphic in a neighborhood of $\partial \Delta$ and for all $\epsilon>0$ small enough,

$$
\left|\int_{\Gamma_{\epsilon}} g(w) h(w) d w\right| \leq C_{g}\|h\|_{\partial \Delta}
$$

where $\Gamma_{\epsilon}=\{w \in \Delta: \operatorname{dist}(w, \partial \Delta)=\epsilon\}$. Then (a) there is a measure $\eta$ which is absolutely continuous with respect to the Lebesgue (arc) measure on $\partial \Delta$ such that $\hat{\eta}$ is $g$ in $\Delta$ and 0 off $\bar{\Delta}$, and (b) we have

$$
\|g\|:=\limsup _{\epsilon \rightarrow 0} \int_{\Gamma_{\epsilon}}|g(w)||d w|<\infty .
$$

Moreover, $\|g\|=\|\eta\|$, the total variation of $\eta$, and there are a sequence $\left\{h_{j}\right\}$ of locally analytic on $\partial \Delta$ functions and a sequence $\epsilon_{j} \rightarrow 0$ such that $\left\|h_{j}\right\|_{\partial \Delta} \rightarrow 1$ as $j \rightarrow \infty$ and

$$
\lim _{j \rightarrow \infty} \int_{\Gamma_{\epsilon_{j}}} g(w) h_{j}(w) d w=\|\eta\|=\|g\| .
$$

Conversely, (11) (obviously) implies 10. 
Proof. 10 means that the conditions of Theorem 2 of Havin are satisfied for the compact $F=\partial \Delta$ and the function which is $g$ in $\Delta$ and 0 outside $\bar{\Delta}$. Hence, there exists a measure $\eta$ supported on $\partial \Delta$ such that $\hat{\eta}=g$ in $\Delta$ and $\hat{\eta}=0$ in $\mathbb{C} \backslash \bar{\Delta}$.

Let us prove (b) first. We can assume that $\partial \Delta$ is the union of $S_{1}=S^{1}$ and finitely many disjoint circles $S_{k}, k=2, \ldots, p$, inside the unit circle $S^{1}$. Let $\eta_{k}=\left.\eta\right|_{S_{k}}, 1 \leq k \leq p$. If $w \in \Delta$ is close to the component $S_{1}=S^{1}$ of $\partial \Delta$, so that $w=r \zeta, \zeta \in S^{1}$, we have

$$
g(r \zeta)=\left(\hat{\eta}_{1}(r \zeta)-\hat{\eta}_{1}(\zeta / r)\right)+\delta(r, \zeta)
$$

where $\delta(r, \zeta)=\sum_{k=2}^{p}\left(\hat{\eta}_{k}(r \zeta)-\hat{\eta}_{k}(\zeta / r)\right)$ tends to 0 uniformly in $\zeta$ as $r \rightarrow 1$ because $\hat{\eta}_{k}$ is analytic off $S_{k}$. Hence, applying Theorem 3 to the measure $\eta_{1}$ we get (11) for a component $\Gamma_{\epsilon}$ which is near $S_{1}$. The proof for the other components of $\Gamma_{\epsilon}$ is very similar. This proves (11).

In turn, (11) means that the condition of Lemma 3.1 is satisfied where $\Omega=\Delta$ (so that $\Delta_{\Omega}=\Delta$ and $\psi_{\Omega}=\mathrm{id}$ ) and $\kappa=g$. By Lemma 3.1, there exists a measure $\nu^{g}$ supported on $\partial \Delta$ and absolutely continuous with respect to the arc measure on $\partial \Delta$ such that $\hat{\nu}^{g}=g$ in $\Delta$ and $\hat{\nu}^{g}=0$ outside of $\bar{\Delta}$. But then the Cauchy transform of the measure $\eta-\nu^{g}$ vanishes outside of $\partial \Delta$. Since $\partial \Delta$ is a C-compact, we conclude that $\nu^{g}=\eta$. This proves part (a) along with $\|\eta\|=\|g\|_{H^{1}}$.

It remains to find a sequence $\left\{h_{j}\right\}$ as in 12 . Note that since $\Delta$ consists of finitely many components (which are circles), it is enough to find $\left\{h_{j}\right\}$ for each component separately. So let

$$
S_{b}(R)=\left\{w=b+R e^{i t}: 0 \leq t \leq 2 \pi\right\}
$$

be such a component. As $g \in H^{1}(\Delta)$ and $g \not \equiv 0$, the non-tangential limit $\tilde{g}$ of $g$ exists and is non-zero for almost every $w \in S_{b}(R)$. Note that for $w \in S_{b}(R),|d w|=\frac{\alpha}{w-b} d w$ where $\alpha=\frac{R}{i}$. Since $\tilde{g} \neq 0$ almost everywhere, the function $H(w)=\frac{\alpha}{w-b} \frac{|\tilde{g}(w)|}{\tilde{g}(w)}$ is in $L^{\infty}\left(S_{b}(R)\right)$. By Luzin's theorem, given $\delta>0$, there is a continuous function $H^{\delta}$ on $S_{b}(R)$ such that $\sup _{w}\left|H^{\delta}(w)\right| \leq$ $\sup _{w}|H(w)|=1$ and $l\left(\left\{w: H(w) \neq H^{\delta}(w)\right\}\right)<\delta$ where $l$ is the Lebesgue (arc) measure on $S_{b}(R)$. In turn, let $h^{\delta}$ be a locally holomorphic function on $S_{b}(R)$ such that $\sup _{w \in S_{b}(R)}\left|H^{\delta}(w)-h^{\delta}(w)\right|<\delta$. Then the sequence of functions $h_{j}:=h^{1 / j}$, defined for all $j$ large enough, and a sequence $\epsilon_{j}$ tending to zero fast enough, work.

Here are the details. Assuming for simplicity $S_{b}(R)=S^{1}$, for each $j \geq 1$ choose $\epsilon_{j}>0$ such that $\left|h^{1 / j}\left(r_{j} e^{i t}\right)-h^{1 / j}\left(e^{i t}\right)\right|<1 / j$ for all $t \in[0,2 \pi]$ and for $r_{j}=1-\epsilon_{j}$. Write

$$
\left|\int_{|w|=r_{j}} g(w) h^{1 / j}(w) d w-\int_{|w|=1}\right| \tilde{g}(w)|| d w|| \leq A_{j}+B_{j}+C_{j}
$$


where

$$
A_{j}:=\left|\int_{|w|=r_{j}} g(w) h^{1 / j}(w) d w-\int_{|w|=1} \tilde{g}(w) h^{1 / j}(w) d w\right| \rightarrow 0
$$

because

$$
\begin{aligned}
A_{j} \leq & \int_{0}^{2 \pi}\left|r_{j} g\left(r_{j} e^{i t}\right)-\tilde{g}\left(e^{i t}\right)\right|\left|h^{1 / j}\left(r_{j} e^{i t}\right)\right| d t \\
& +\int_{0}^{2 \pi}\left|\tilde{g}\left(e^{i t}\right)\right|\left|h^{1 / j}\left(r_{j} e^{i t}\right)-h^{1 / j}\left(e^{i t}\right)\right| d t \rightarrow 0
\end{aligned}
$$

as $\left.\int_{0}^{2 \pi} \mid g\left(r e^{i t}\right)-\tilde{g}\left(e^{i t}\right)\right) \mid d t \rightarrow 0$ with $r \rightarrow 1$ and by the choice of $\epsilon_{j}$,

$$
B_{j}:=\left|\int_{|w|=1}\left(\tilde{g}(w) h^{1 / j}(w)-\tilde{g}(w) H^{1 / j}(w)\right) d w\right| \rightarrow 0
$$

as $\sup _{|w|=1}\left|H^{1 / j}(w)-h^{1 / j}(w)\right| \rightarrow 0$ as $j \rightarrow \infty$, and

$$
\begin{aligned}
C_{j} & :=\left|\int_{|w|=1} \tilde{g}(w) H^{1 / j}(w) d w-\int_{|w|=1}\right| \tilde{g}(w)|| d w|| \\
& =\left|\int_{|w|=1}\left(\tilde{g}(w) H^{1 / j}(w)-\tilde{g}(w) H(w)\right) d w\right| \rightarrow 0
\end{aligned}
$$

as $l\left(\left\{w: H(w) \neq H^{1 / j}(w)\right\}\right) \rightarrow 0$ as $j \rightarrow \infty$.

Another result we are going to use belongs to approximation theory. Note that we do not use it in full generality - see comments right after the statement.

Theorem 4 (A. Davie [7], 8], Zhijian Qiu [20]). Let $U$ be a bounded open subset of $\mathbb{C}$ such that each of its components is finitely connected and the complement of $U$ contains no isolated points. Then the following conditions are equivalent:

(i) the collection of components of $U$ is a D-collection,

(ii) $A(U)$ is strongly boundedly pointwise dense in $H^{\infty}(U)$ : each bounded analytic function $h$ on $U$ is a pointwise limit of a sequence $h_{n} \in A(U)$ with $\left\|h_{n}\right\|_{\bar{U}} \leq\|h\|_{U}$.

Here are some comments on Theorem 4 and the way we apply it:

(1) We need the implication (i) $\Rightarrow$ (ii) only.

(2) By Davie [8], (ii) is equivalent to a seemingly weaker statement (ii'): each bounded analytic function $h$ on $U$ is a pointwise limit of a bounded sequence $\left\{h_{n}\right\} \subset A(U)$.

(3) Davie [7] (see preceding [6] though) proved that (i) and (ii') are equivalent when every component of $U$ is simply connected. 
(4) The case when all but finitely many components of $U$ are simply connected can be reduced easily to that in (3) with the help of a simple geometric construction (by covering every finitely connected component by finitely many simply connected ones using only inner smooth cuts and then applying a local criterion of [1] for $A(U)$ to be pointwise boundedly dense in $H^{\infty}(U)$; see [20, Lemma 2.1] for the details).

(5) We employ Theorem 4 only to functions $h$ which are non-zero on finitely many components $U_{1}, \ldots, U_{m}$ of $U$. This case can be reduced to the case (4) as follows. Firstly, since $h=\sum_{i=1}^{m} h \chi_{U_{i}}$, it is enough to prove the claim for each $h \chi_{U_{i}}$ separately, i.e., when $h$ is non-zero only on a single component, say $U_{1}$. Now, if $U_{2}, U_{3}, \ldots$ are all other components of $U$, let us modify $U$ to get a larger open set $\tilde{U}$ roughly by joining to each $U_{j}(j>1)$ some components of $\mathbb{C} \backslash U_{j}$ disjoint from $U_{1}$ to turn it into a simply connected domain; see details in [20, proof of Theorem 2.1]. Then $h \chi_{U_{1}}$ is bounded analytic in $\tilde{U}$ and $A(\tilde{U}) \subset A(U)$, and we apply the case (4) to $\tilde{U}$.

We need the following consequence of Theorem 4 .

Corollary 3.1. Let $V$ and $\left(\Omega_{i}\right)_{i=1}^{N}$ be as in $(\mathrm{P} 1)$ of Theorem 1, i.e., conditions (I)-(II) hold and $\bar{V}$ is an A-compact. Fix $1 \leq m \leq N$. Given $\epsilon>0$ small enough, let $h$ be a bounded analytic function in $D_{\epsilon}:=\bigcup_{k=1}^{m} \Omega_{k} \backslash$ $\overline{\Omega_{k, \epsilon}}$. Then given a positive sequence $\sigma_{n} \rightarrow 0$, there is a sequence of rational functions $R_{n}$ with poles outside of $E_{\epsilon}:=\bar{V} \backslash \bigcup_{k=1}^{m} \Omega_{k, \epsilon}$ such that $\left\|R_{n}\right\|_{E_{\epsilon}} \leq$ $\|h\|_{D_{\epsilon}}+\sigma_{n}, R_{n}(z) \rightarrow h(z)$ for every $z \in D_{\epsilon}$ and $R_{n} \rightarrow 0$ in $V \backslash \bigcup_{k=1}^{m} \bar{\Omega}_{k}$.

Proof. Since $\bar{V}$ is an A-compact and the boundary of $E_{\epsilon}$ is the disjoint union of $\partial V$ and finitely many analytic curves $\partial \Omega_{k, \epsilon}, 1 \leq k \leq m, E_{\epsilon}$ is an A-compact as well. This follows e.g. from Vitushkin's theorem [22]. Now, given $\epsilon>0,\left\{\sigma_{n}\right\}$ and $h$ as in the statement, let us extend $h$ from $D_{\epsilon}$ to a (bounded analytic) function $h^{1}$ in $V_{\epsilon}:=\bigcup_{k=1}^{N} \Omega_{k} \backslash \bigcup_{k=1}^{m} \overline{\Omega_{k, \epsilon}}$ by defining $h^{1}=0$ on $\bigcup_{k=m+1}^{N} \Omega_{k}$. In view of condition (I), by Theorem 4 , there is a sequence $h_{n} \in A\left(V_{\epsilon}\right)$ such that $h_{n}(z) \rightarrow h^{1}(z)$ as $n \rightarrow \infty$ for all $z \in V_{\epsilon}$ and $\left\|h_{n}\right\|_{V_{\epsilon}} \leq\left\|h^{1}\right\|_{V_{\epsilon}}=\|h\|_{D_{\epsilon}}$. By condition (II), $h_{n} \in A\left(\bar{V}_{\epsilon}\right)=A\left(E_{\epsilon}\right)$. Since $E_{\epsilon}$ is an A-compact, for each $n$ there is a rational function $R_{n}$ with poles outside of $E_{\epsilon}$ such that $\left\|R_{n}-h_{n}\right\|_{E_{\epsilon}}<\sigma_{n}$.

The sequence $R_{n}$ is as required. Indeed, for any $z \in E_{\epsilon}$,

$$
\left|R_{n}(z)-h^{1}(z)\right| \leq\left|R_{n}(z)-h_{n}(z)\right|+\left|h_{n}(z)-h^{1}(z)\right|<\sigma_{n}+\left|h_{n}(z)-h^{1}(z)\right|,
$$

hence $\left\{R_{n}\right\}$ tends to $h^{1}(z)=h(z)$ on $D_{\epsilon}$ and to $h^{1}(z)=0$ on $\bigcup_{k=m+1}^{N} \Omega_{k}$. At the same time, $\left\|R_{n}\right\|_{E_{\epsilon}} \leq\left\|h_{n}\right\|_{E_{\epsilon}}+\sigma_{n} \leq\|h\|_{D_{\epsilon}}+\sigma_{n}$.

3.2. Proof of Theorem 1. Let us start with the more difficult part (P1): assume that there is a measure $\nu$ on $E$ such that (1) holds and prove (b). First of all, since $\hat{\nu}=0$ off $E \cup \bar{V}$ and $E$ is a C-compact by 
condition (III)(ii), Lemma 2.1 immediately tells us that $\nu=0$ on $E \backslash \partial V$. In other words, one can assume from the beginning that

$$
E=\partial V \text {. }
$$

Note at this point that since $\bar{V}$ is an A-compact by (III)(i), its boundary $E$ is a C-compact (see [10, p. 227]).

Now, let $\tilde{C}=C_{g}$ be the constant guaranteed by Theorem 2 for the compact $E=\partial V$ and the function $g=g_{\kappa}$ where

$$
g_{\kappa}(z)= \begin{cases}\kappa_{i}(z) & \text { if } z \in \Omega_{i}, \text { for each } i, \\ 0 & \text { if } z \in \mathbb{C} \backslash \bar{V} .\end{cases}
$$

Let us fix a collection of uniformizations $\psi_{\Omega_{k}}: \Delta_{\Omega_{k}} \rightarrow \Omega_{k}$, where the closures of the circular domains $\Delta_{\Omega_{k}}$ are pairwise disjoint. Write $\psi_{k}=\psi_{\Omega_{k}}$ and $\Delta_{k}=\Delta_{\Omega_{k}}$. Let

$$
\tilde{\kappa}_{k}(w)=\kappa_{k}\left(\psi_{k}(w)\right) \psi_{k}^{\prime}(w)
$$

for $w \in \Delta_{k}$. Fix $1 \leq m \leq N$.

Claim. For every function $\tilde{h}$ which is holomorphic in a small enough neighborhood of $\Gamma:=\bigcup_{k=1}^{m} \partial \Delta_{k}$ and every $\epsilon>0$ small enough,

$$
\left|\sum_{k=1}^{m} \int_{\Gamma_{k, \epsilon}} \tilde{\kappa}_{k}(w) \tilde{h}(w) d w\right| \leq \tilde{C}\|\tilde{h}\|_{\Gamma}
$$

where $\Gamma_{k, \epsilon}=\left\{w \in \Delta_{k}: \operatorname{dist}\left(w, \partial \Delta_{k}\right)=\epsilon\right\}$.

Proof of the Claim. Fix any $\epsilon>0$ such that $\tilde{h}$ is holomorphic in a neighborhood of $\bigcup_{k=1}^{m}\left\{w \in \Delta_{k}: \operatorname{dist}\left(w, \partial \Delta_{k}\right) \leq \epsilon\right\}$. Then, for each $\tilde{\epsilon} \in(0, \epsilon)$ and $k \in\{1, \ldots, m\}$,

$$
\int_{\Gamma_{\tilde{\epsilon}}} \tilde{\kappa}_{k}(w) \tilde{h}(w) d w=\int_{\Gamma_{\epsilon}} \tilde{\kappa}_{k}(w) \tilde{h}(w) d w .
$$

Let $h=\tilde{h} \circ \phi_{k}$ where $\phi_{k}=\psi_{k}^{-1}: \Omega_{k} \rightarrow \Delta_{k}$. Let $\epsilon_{0} \in(\tilde{\epsilon}, \epsilon)$. Then $h$ is defined in $D_{\epsilon_{0}}=\bigcup_{k=1}^{m} \Omega_{k} \backslash \bar{\Omega}_{k, \epsilon_{0}}$. Moreover, $h$ is holomorphic and bounded in $D_{\epsilon_{0}}$. Let $E_{\epsilon_{0}}=\bar{V} \backslash \bigcup_{k=1}^{m} \Omega_{k, \epsilon_{0}}$. Fix a positive sequence $\sigma_{n} \rightarrow 0$ and, by Corollary 3.1, find a sequence of rational functions $R_{n}$ with poles outside $E_{\epsilon_{0}}$ such that $R_{n}(z) \rightarrow h(z)$ for all $z \in D_{\epsilon_{0}}, R_{n}(z) \rightarrow 0$ for all $z \in \bigcup_{k=m+1}^{N} \Omega_{k}$ and $\left\|R_{n}\right\|_{E_{\epsilon_{0}}} \leq\|h\|_{D_{\epsilon_{0}}}+\sigma_{n}$ for all $n$. Hence, for $k=1, \ldots, m$ and fixed $\tilde{\epsilon}$,

$$
\int_{\Gamma_{\tilde{\epsilon}}} \tilde{\kappa}_{k}(w) \tilde{h}(w) d w=\int_{\partial \Omega_{k, \tilde{\epsilon}}} \kappa_{k}(z) h(z) d z=\lim _{n \rightarrow \infty} \int_{\partial \Omega_{k, \tilde{\epsilon}}} \kappa_{k}(z) R_{n}(z) d z .
$$

Consider a finite collection $A_{\tilde{\epsilon}}:=\left\{\partial \Omega_{k, \tilde{\epsilon}}\right\}_{k=1}^{m}$ of closed analytic curves. Let us complete it by a finite collection $B_{\tilde{\epsilon}}$ of pairwise disjoint closed analytic curves in $\mathbb{C} \backslash \bigcup_{k=1}^{m} \bar{\Omega}_{k}$ such that altogether $A_{\tilde{\epsilon}} \cup B_{\tilde{\epsilon}}=\partial U_{\tilde{\epsilon}}$ where $U_{\tilde{\epsilon}}$ is a (small) 
neighborhood of $E$. Note that $R_{n} \rightarrow h$ on $A_{\tilde{\epsilon}}, R_{n} \rightarrow 0$ on $B_{\tilde{\epsilon}} \cap \bigcup_{k=m+1}^{N} \Omega_{k}$ while $\hat{\nu}=0$ on $B_{\tilde{\epsilon}} \cap(\mathbb{C} \backslash \bar{V})$. Therefore, by the choice of $\tilde{C}$,

$$
\begin{aligned}
& \lim _{n \rightarrow \infty}\left|\sum_{k=1}^{m} \int_{\partial \Omega_{k, \tilde{\epsilon}}} \kappa_{k}(z) R_{n}(z) d z\right| \\
& \quad=\lim _{n \rightarrow \infty}\left|\int_{\partial U_{\tilde{\epsilon}}} g_{\kappa}(z) R_{n}(z) d z\right| \leq \limsup _{n} \tilde{C}\left\|R_{n}\right\|_{E_{\epsilon_{0}}} \\
& \quad \leq \lim _{n} \tilde{C}\left(\|h\|_{D_{\epsilon_{0}}}+\sigma_{n}\right)=\tilde{C}\|h\|_{D_{\epsilon_{0}}}=\tilde{C}\|\tilde{h}\|_{\bigcup_{k=1}^{m}\left\{w \in \Delta_{k}: \operatorname{dist}\left(w, \partial \Delta_{k}\right) \leq \epsilon_{0}\right\}} .
\end{aligned}
$$

Thus, by the latter inequality along with 15 and (16), for every $\epsilon_{0} \in(0, \epsilon)$,

$$
\left|\sum_{k=1}^{m} \int_{\Gamma_{\epsilon}} \tilde{\kappa}_{k}(w) \tilde{h}(w) d w\right| \leq \tilde{C}\|\tilde{h}\|_{\bigcup_{k=1}^{m}\left\{w \in \Delta_{k}: \operatorname{dist}\left(w, \partial \Delta_{k}\right) \leq \epsilon_{0}\right\}} .
$$

But $\lim _{\epsilon_{0} \rightarrow 0}\|\tilde{h}\|_{\left\{w \in \Delta_{h}: \operatorname{dist}\left(w, \partial \Delta_{k}\right) \leq \epsilon_{0}\right\}}=\|\tilde{h}\|_{\partial \Delta_{k}}$ because $\tilde{h}$ is continuous up to $\partial \Delta_{k}$. This proves (14) and the Claim.

We now proceed as follows. Since the closures of $\Delta_{k}, 1 \leq k \leq m$ (where $m$ is finite) are pairwise disjoint, given $k \in\{1, \ldots, m\}$, the Claim immediately implies that the condition of Lemma 3.2 holds for $\Delta=\Delta_{k}$ and $g=\tilde{\kappa}_{k}(w)$. We conclude there exist a measure $\eta_{k}$ which is absolutely continuous with respect to the Lebesgue measure on $\partial \Delta_{k}$, a sequence $\left\{h_{k, j}\right\}_{j=1}^{\infty}$ of functions locally analytic near $\partial \Delta_{k}$ and a sequence $\epsilon_{j} \rightarrow 0$ such that $\left\|h_{k, j}\right\|_{\partial \Delta_{k}} \rightarrow 1$ as $j \rightarrow \infty$ and

$$
\lim _{j \rightarrow \infty} \int_{\Gamma_{k, \epsilon_{j}}} \kappa_{k}\left(\psi_{k}(w)\right) \psi_{k}^{\prime}(w) h_{k, j}(w) d w=\left\|\eta_{k}\right\|=\left\|\kappa_{k}\right\|,
$$

where $\left\|\kappa_{k}\right\|=\lim \sup _{\epsilon \rightarrow 0} \int_{\partial \Omega_{k, \epsilon}}\left|\kappa_{k}(z)\right||d z|$. Now, apply the Claim with $\tilde{h}_{j}$ being $\tilde{h}_{k, j}$ near $\partial \Delta_{k}, 1 \leq k \leq m$. By Lemma 3.2 and the Claim,

$$
\sum_{k=1}^{m}\left\|\kappa_{k}\right\|=\sum_{k=1}^{m}\left\|\eta_{k}\right\|=\sum_{k=1}^{m} \limsup _{\epsilon \rightarrow 0} \int_{\Gamma_{k, \epsilon}}\left|\kappa_{k}\left(\psi_{k}(w)\right) \psi_{k}^{\prime}(w)\right||d w| \leq \tilde{C} .
$$

Since $\tilde{C}$ is independent of $m$, this proves (3), that is, $\sum_{k=1}^{N}\left\|\kappa_{k}\right\|<\infty$. This allows us to easily finish the proof that (a) implies (b) as follows. For each $1 \leq k \leq N$, by Lemma 3.1 (with $\Omega=\Omega_{k}$ and $\kappa=\kappa_{k}$ ) there is a measure $\nu^{\kappa_{k}}$ such that $\hat{\nu}^{\kappa_{k}}=\kappa_{k}$ in $\Omega_{k}$ and $\hat{\nu}^{\kappa_{k}}=0$ off $\overline{\Omega_{k}}$, and moreover $\nu^{\kappa_{k}} \ll \omega_{\Omega_{k}}$ and $\left\|\nu_{\kappa_{k}}\right\|=\left\|\kappa_{k}\right\|$. Define $\nu_{w}=\sum_{k=1}^{N} \nu^{\kappa_{k}}$. Then $\left\|\nu_{w}\right\|=\sum_{k=1}^{N}\left\|\nu^{\kappa_{k}}\right\|<\infty$. Since by condition (I) harmonic measures of different $\Omega_{k}$ are pairwise singular, $\left\{\nu^{\kappa_{k}}\right\}_{k=1}^{N}$ are pairwise singular as well. Now, $\hat{\nu}_{w}=\sum_{k=1}^{N} \hat{\nu}^{\kappa_{k}}$ is equal to $\kappa_{k}$ in $\Omega_{k}$ for each $k$, and 0 off $\bar{V}$. Let us compare the measures $\nu$ and $\nu_{\omega}$. For the difference measure $\tau=\nu-\nu_{\omega}$, we have $\hat{\tau}=0$ off $E$ where $E$ is a 
C-compact. Hence, $\tau=\nu-\nu_{\omega}=0$, and $(\mathrm{a}) \Rightarrow(\mathrm{b})$ is proved. In fact, the above considerations also prove (P2), by using again Lemma 3.1.

Acknowledgements. We would like to thank Alexander Volberg for communicating Example 1 and for useful comments, and the referee for the reference [16]. The author acknowledges the support of ISF Grant No. $1226 / 17$.

\section{References}

[1] C. J. Bishop, A characterization of Poissonian domains, Ark. Mat. 29 (1991), 1-24.

[2] C. J. Bishop, L. Carleson, J. B. Garnett and P. W. Jones, Harmonic measures supported on curves, Pacific J. Math. 138 (1989), 233-236.

[3] C. J. Bishop, Constructing continuous functions holomorphic off a curve, J. Funct. Anal. 82 (1989), 113-137.

[4] E. Bishop, The structure of certain measures, Duke Math. J. 25 (1958), 283-289.

[5] E. Bishop, Boundary measures of analytic differentials, Duke Math. J. 27 (1960), 331-340.

[6] A. Browder and J. Wermer, Some algebras of functions on an arc, J. Math. Mech. 12 (1963), 119-130.

[7] A. M. Davie, Dirichlet algebras of analytic functions, J. Funct. Anal. 6 (1970), 348356.

[8] A. M. Davie, Bounded approximation and Dirichlet sets, J. Funct. Anal. 6 (1970), 460-467.

[9] E. P. Dolzhenko, On approximation on closed regions and on null-sets, Dokl. Akad. Nauk SSSR 143 (1962), 771-774 (in Russian).

[10] T. Gamelin, Uniform Algebras, Prentice-Hall, 1969.

[11] T. Gamelin and J. Garnett, Constructive techniques in rational approximation, Amer. J. Math. 89 (1967), 932-941.

[12] J. Garnett, Analytic Capacity and Measure, Lecture Notes in Math. 297, Springer, 1972.

[13] L. Glicksberg, A remark on analyticity of function algebras, Pacific J. Math. 13 (1963), 1181-1185.

[14] S. Fisher, Function Theory on Planar Domains, Wiley, 1983.

[15] D. Khavinson, Annihilating measures of the algebra $R(X)$, J. Funct. Anal. 58 (1984), 175-193.

[16] D. Khavinson, F. and M. Riesz Theorem, analytic balayage, and problems in rational approximation, Constr. Approx. 4 (1988), 341-356.

[17] P. Koosis, Introduction to $H_{p}$ Spaces, Cambridge Univ. Press, Cambridge, 1980.

[18] G. Levin, Fixed points of the Ruelle-Thurston operator and the Cauchy transform, arXiv:2002.03430 (2020).

[19] Ch. Pommerenke and B. Rodin, Intrinsic rotations of simply connected regions, II, Complex Var. Theory Appl. 4 (1985), 223-232.

[20] Z. Qiu, On pointwise bounded approximation, Acta Math. Sinica (English Ser.) 25 (2009), 1217-1222.

[21] G. G. Tumarkin, On integrals of Cauchy-Stieltjes type, Uspekhi Mat. Nauk (N.S.) 11 (1956), 163-166 (in Russian). 
[22] A. G. Vitushkin, Analytic capacity of sets in problems of approximation theory, Russian Math. Surveys 22 (1967), 139-200.

[23] L. Zalcman, Analytic Capacity and Rational Approximation, Lecture Notes in Math. 50, Springer, 1968.

Genadi Levin

Institute of Mathematics

Hebrew University of Jerusalem

Givat Ram, Jerusalem, Israel

E-mail: levin@math.huji.ac.il 